\title{
TOPSAT - HIGH RESOLUTION IMAGING FROM A SMALL SATELLITE
}

\author{
Paul Brooks \\ Space Department \\ 1004, A8 Building, QinetiQ Farnborough \\ UK, GU14 OLX
}

Tel: +44 1252393267

Fax: +44 1252396320

Email:pbrooks@scs.dera.gov.uk

SESSION 3: NATIONAL NEEDS AND OBJECTIVES

\begin{abstract}
$\underline{\text { Abstract }}$
The Topsat mission is designed to demonstrate the capabilities of small satellites for classically high value remote sensing missions. Conceived by the UK's Defence Evaluation and Research Agency (DERA), it will produce $2.5 \mathrm{~m}$ resolution panchromatic imagery and $5 \mathrm{~m}$ resolution multispectral imagery in three visible wavebands and allow direct transmission of the image data to a local mobile ground station.

The Topsat programme is jointly funded by the UK Ministry of Defence (MoD) and by the British National Space Centre's Mosaic small satellite initiative and includes provision for the spacecraft, ground segment, commercial launch and one year of planned operations.

The mission will meet the national needs of the UK in two areas. The UK MoD will use Topsat to investigate the utility and issues of providing data acquisition and tasking resources to local command levels. BNSC are supporting the programme as a demonstration of an enhanced capability for UK small satellites leading to sales of similar systems and to allow the development of lower cost markets for remote sensing products producible from such systems.

The Topsat mission is a collaboration between four UK partners. DERA are leading the mission and providing the data handling unit, high rate downlink, mobile ground station and operations. Surrey Satellites (SSTL) are providing the spacecraft bus and command and telemetry links. Rutherford Appleton Laboratory (RAL) are developing a near diffraction limited wide field of view camera which, when combined with a pitch compensation manoeuvre, allows the highly demanding optical performance characteristics to be achieved. Infoterra are responsible for developing potential markets for Topsat and future derivatives.
\end{abstract}




\section{Introduction}

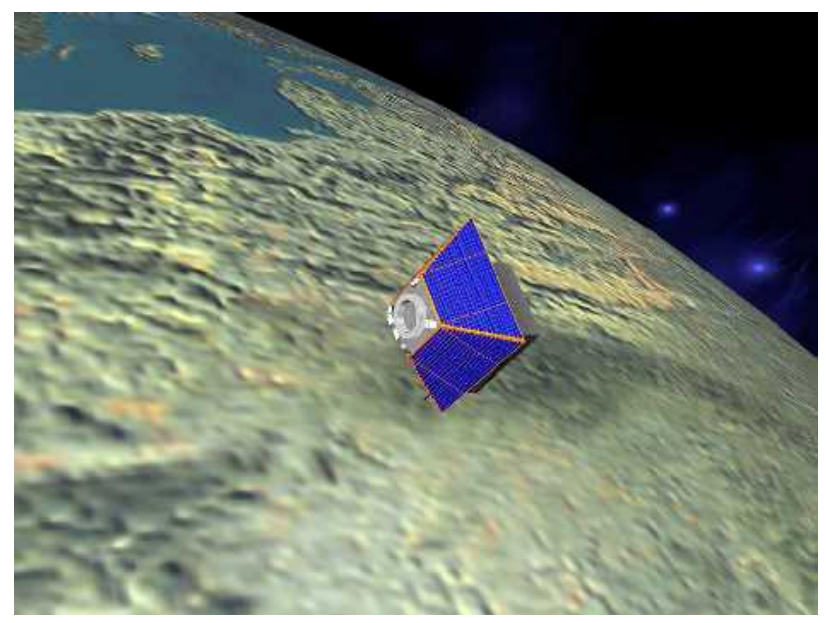

Topsat is an end to end high quality imaging system based on a small satellite with direct user access from transportable ground facilities. The system is designed to meet three objectives critical to the future of small satellite systems:

- To increase the sales of small satellites and maintain a competitive manufacturing base in the UK

- To demonstrate the use of low cost satellites to provide information direct to users in the field in response to their tasking requests

- To support the development of new markets for low cost, high quality image and information products with excellent timeliness

Further, for the UK MoD, Topsat will enable the practical implications of the use of space based surveillance information to be investigated as well as providing a data source to trial different architectures for information management.

These objectives will be met by an agile, small satellite in an end-to-end mission. It will use an innovative, compact optical system providing $2.5 \mathrm{~m}$ resolution panchromatic and $5 \mathrm{~m}$ multispectral imagery over a wide swath, together with a downlink direct to local users. The programme is funded by the UK Ministry of Defence (MoD) and the British National Space Centre (BNSC) Mosaic small satellite initiative and includes provision for the spacecraft, ground segment, commercial launch and one year of planned demonstration.

Topsat was conceived by the UK's Defence Evaluation and Research Agency (DERA) Space Department as an experiment to test the capabilities of low cost satellites to provide useful remote sensing data. The original remit was to limit the cost of a system to under $£ 10 \mathrm{M}$ and determine the level of performance that may be achievable with such a constraint. The constraint led to the consideration of commercial parts, novel techniques and technologies and the tight restriction of user requirements.

The self-imposed constraint led to three significant features of the Topsat system,

- the development by RAL of a novel 3 mirror off-axis camera design to maximise the available light from a small aperture and minimise the contrast degradation formed by obscuring elements

- the definition of a simple pitch compensation manoeuvre to increase the effective integration time of the imaging sequence

- the utilisation of the small satellite technology of SSTL and DERA to minimise the cost of the system

These features have led to an intriguing balance of innovation, risk and cost that requires an in-orbit demonstration of capability. 


\section{Topsat System}

Topsat has been developed from an initial assessment of what would be the highest resolution reasonably achievable from a small satellite (c.100kg) platform. This initial assessment led to the basis of a $2.5 \mathrm{~m}$ panchromatic mission using pitch compensation time delayed integration (TDI) to compensate for the insufficient light levels from most areas of the world, most of the year.

The basis for the next level of design was to maintain as simple a system as possible consistent with a limited baseline set of requirements. Specifically it was intended to maintain a platform design with the minimum sources of dynamic noise to facilitate a simple and therefore cost effective approach to the pitch compensation and demanding attitude requirements of a $2.5 \mathrm{~m}$ imaging system. By not including sources of disturbance such as deployable arrays and antennae, fuel and pointing mechanisms it would be possible to relax significantly the requirement on boresight pointing control and knowledge. This approach has been developed through simulation but a flight demonstration is required to validate the assumptions and models.

Removal of disturbance sources naturally leads to significant constraints that are compatible with small satellite techniques. Solar arrays must be body mounted leading to a tight constraint on available power in a wellilluminated (solar beta angle $<30$ degrees) sun synchronous orbit. To counter this problem SSTL have developed a novel truncated spacecraft structure to maximise the aspect of body mounted panels and optimise the use of fairing volume for multiple deployment launches. The removal of pointing mechanisms requires a rigid body motion of the satellite to provide the pointing and pitch compensation TDI. To allow a reasonable response time requires a low overall inertia - again consistent with small satellites.
Whilst the original plan for Topsat was to use an adapted commercial telescope, it was determined at an early stage that developing systems of high effectiveness with simple pointing required the capability to image a large field of view. This in turn led to the consideration of an off-axis system capable of providing the wide field of view in the single axis required of a push-broom system. Whilst this was a considerable development it was considered that within the scope of the complete programme such a camera would provide a better balance of risk and innovation.

The final critical aspect of the system design was the need to be able to undertake local operations and data dissemination. Clearly with a single satellite it is not possible to demonstrate the timeliness available from a constellation but it is required to determine those aspects which might critically affect the utility of high timeliness systems that can not be simulated through orbit and constellation modelling. When the options for command and data dissemination architectures were studied it became clear that the critical technical requirement was for a direct local downlink of the high bandwidth imagery data. Within the constraints of a small satellite already providing such a performant imaging capability, it was not considered viable to provide an additional direct command access. Therefore it was decided to provide a downlink capable of delivering a full image to a local, mobile ground station on the same pass.

With these critical aspects of the system design outlined it is appropriate to consider each aspect in more detail. 


\section{Spacecraft}

The spacecraft bus is being developed and built by SSTL. The current design is currently being developed as a structural qualification model (SQM), ready for qualification in August 2001. The subsystems utilise the successful heritage of SSTL spacecraft to minimise risks in most areas with the structure and attitude control system being the only significant changes from previous missions.

\section{Structure}

The spacecraft utilises a payload plate to provide a simple interface between bus systems and payload. The payload plate is supported by a thrust tube comprising of the SSTL equipment stack. The payload plate accommodates the camera, data handling unit and $\mathrm{x}$ band transmission systems. The design currently provides for a $44 \mathrm{~kg}$ payload mass within a $110 \mathrm{~kg}$ total spacecraft mass.

\section{Attitude dynamics and control}

Attitude will be maintained by reaction wheel control in three axes, with momentum being offloaded through magnetorquers. Earth and sun sensors will maintain Earth pointing whilst a high precision 3-axis gyro will allow the initialisation of the imaging manoeuvre and image rectification if required.

The use of a small rigid spacecraft allows simpler control algorithms to be used and the possibility of open loop control to be considered. DERA have undertaken the initial simulation of the spacecraft to determine the viability of meeting the imaging requirements with the simple sensors, actuators and control algorithms already available for small satellites. The results of using a single roll offset to image off-track, a single yaw offset to correct for the relative ground motion of the boresight and a constant pitch rate to provide the TDI is shown in Fig 1. The spacecraft pointing behaviour associated with this manoeuvre is shown in Fig 2.

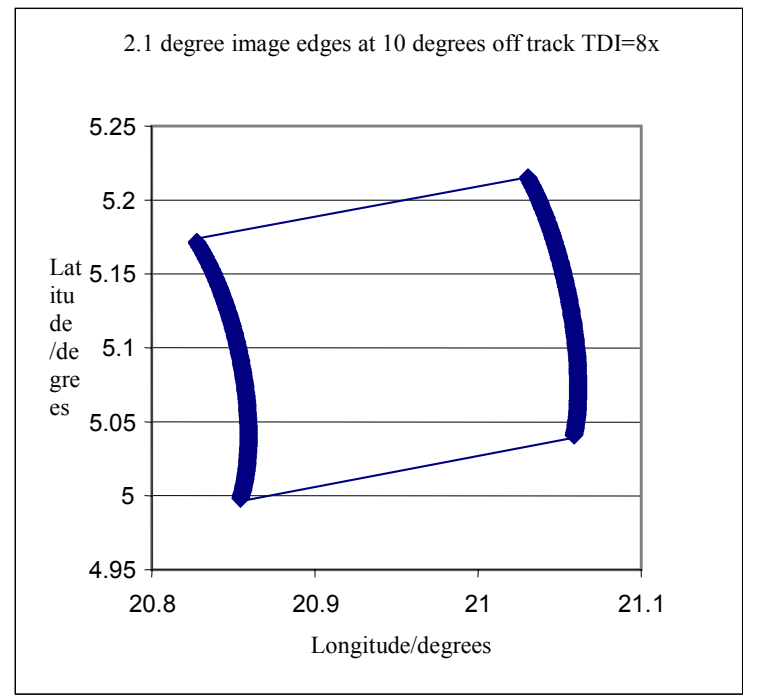

Fig 1 Simulation of effect on image of simple TDI manoeuvre

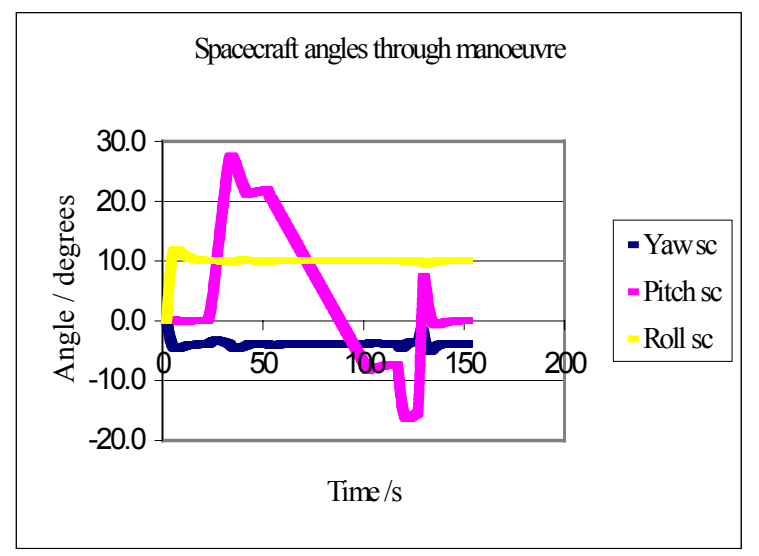

Fig 2 Spacecraft angles through TDI manoeuvre

Even with the highly demanding off-axis and TDI rate requested $(8$ times compensation) the simple manoeuvre provides a viable image in terms of the orthogonality between image lines and the track of the boresight.

\section{Power}

Three body-mounted arrays of GaAs cells will provide $42 \mathrm{~W}$ orbital average power with 96Whrs of power storage in $\mathrm{NiCd}$ batteries. This is sufficient to provide one image and downlink opportunity every other orbit, including an archive link to a polar ground station. 


\section{Communications}

The SSTL microsatellite bus employs standard VHF and UHF communications subsystems. The VHF uplink receivers will be used for telecommanding the on board computer (OBC) software and for uplink file transfer. Similarly the UHF downlink will be used for telemetry, OBC downlink and downlink file transfer.

\section{Command and Data Handling}

This will use the 80386 processor architecture as proven on previous SSTL satellites. It will provide semiautonomous control of the satellite, but it will also include safe modes for initial acquisition or operation during anomalous periods. All of these operations will be supported by flight-proven software running under a multitasking operating system.

\section{Camera}

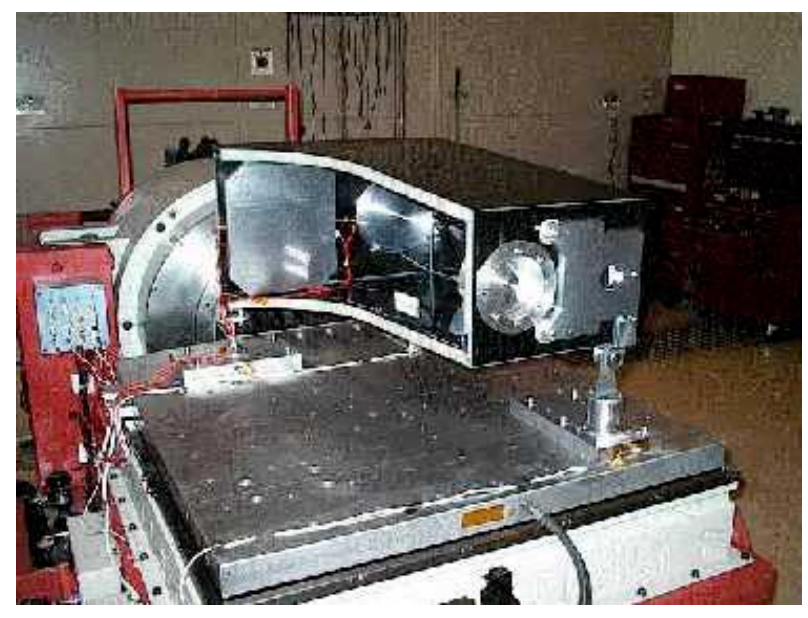

Fig 3 Engineering model undergoing vibration testing

(Courtesy RAL 2001)

The optical system has a focal length of 1680$\mathrm{mm}$. and a collecting aperture of 200-mm. diameter. It comprises three mirrors, the optical surface of each of which is an aspheric of conic form, designed so that the system gives a flat image, free of first-order aberrations. Two of the mirrors are off-axis sections so that there is no central obstruction in the aperture of the system. The design provides a nearly diffraction-limited field of view that is wide in the across-track direction and sufficiently wide in the alongtrack direction to accommodate the number of detector arrays required. The support structure for the mirrors and detector will be fabricated from Carbon Fibre Reinforced Polymer (CFRP) panels, bonded together to form a lightweight, stiff structure. The focal plane assembly will comprise two separate charge coupled device (CCD) detector packages to provide panchromatic imaging at $2.5 \mathrm{~m}$ GSD (ground sampling distance) and 3 -colour imaging at $5 \mathrm{~m}$ resolution.

\section{Data handling and downlink}

600 Mbits of image data will be produced at each acquisition. For the purposes of the local operation demonstration it is required that this data can be downlinked to a mobile $2.5 \mathrm{~m}$ antenna within the local area of the image. DERA are providing the data handling unit, capable of receiving and formatting and storing up to four data sets, an X-band downlink capable of providing $25 \mathrm{Mbs}^{-1}$ and the BURS "Rapid" based mobile ground station.

It should be noted that, for the single satellite demonstrator, the provision of local data transfer is likely to be limited to specific trials to meet the objectives of the military and civil end users due to the limited occasions at which the downlink would be available. This requires the capability to store images for a later archive downlink to a fixed ground station. The data handling unit will cater for this by storing up to four images in permanent memory, thus allowing long term storage without maintaining a high power demand.

The $\mathrm{X}$ band antenna has been designed to flatten the gain across the beam, thus allowing a usable gain at off axis angles of $60^{\circ}$. This provides the capability to downlink the data to a ground terminal significantly off track from the target image area without further manoeuvring of the spacecraft. A 
compact crossed dipole design has already been built and characterised at DERA.

Currently the operational utility of the complete system is limited by the RAPIDS mobile antenna which has a constricted range of elevation and azimuth due to the low cost hydraulic ram system employed. The probability of being able to image an area around the mobile ground segment based in Farnborough, UK and downlink it in the same pass is shown in Fig 5. This shows the effect of combining the off-track capability of the satellite, the 60 degree limit on the $\mathrm{X}$ band antenna and the restriction on the elevation and azimuth capability of the mobile antenna.

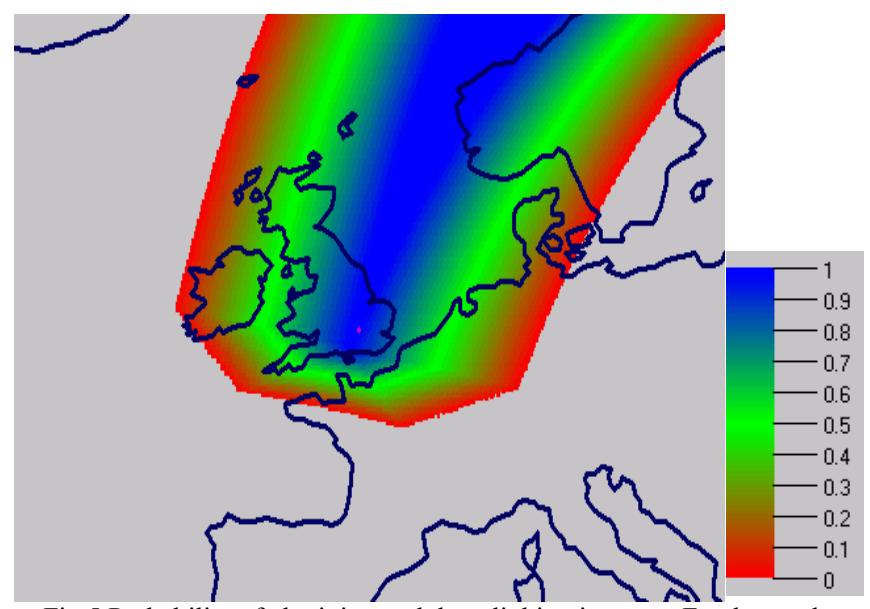

Fig 5 Probability of obtaining and downlinking image to Farnborough

\section{Operations}

Tasks will be provided by the DERA operations team to the SSTL ground station in Guildford where a nominal 3 days worth of commands will be uploaded on a daily basis. Whilst it is intended that the system be capable of providing one image per orbit, in practice it is expected that this will be limited by appropriate tasks to approximately 5 images per day. The local operator of the system will be able to undertake initial planning of operations and place a task on the system through a simple link to the SSTL ground station.
A series of technical tests will be undertaken in addition to the specific exercise required by the MoD sponsors. Additional it is intended to allow potential civil and commercial users access to the system to test and develop the novel marketing strategies, currently under development by Infoterra, that are allowed by such a low cost and flexible system.

The mobile ground segment required for local operations has already been developed from the RAPIDS system. This is now complete, as shown in Fig 6, with a fully integrated, mobile and robust system being provided by a Landrover, fitted with the planning and processing equipment, being capable of towing the antenna to the site.
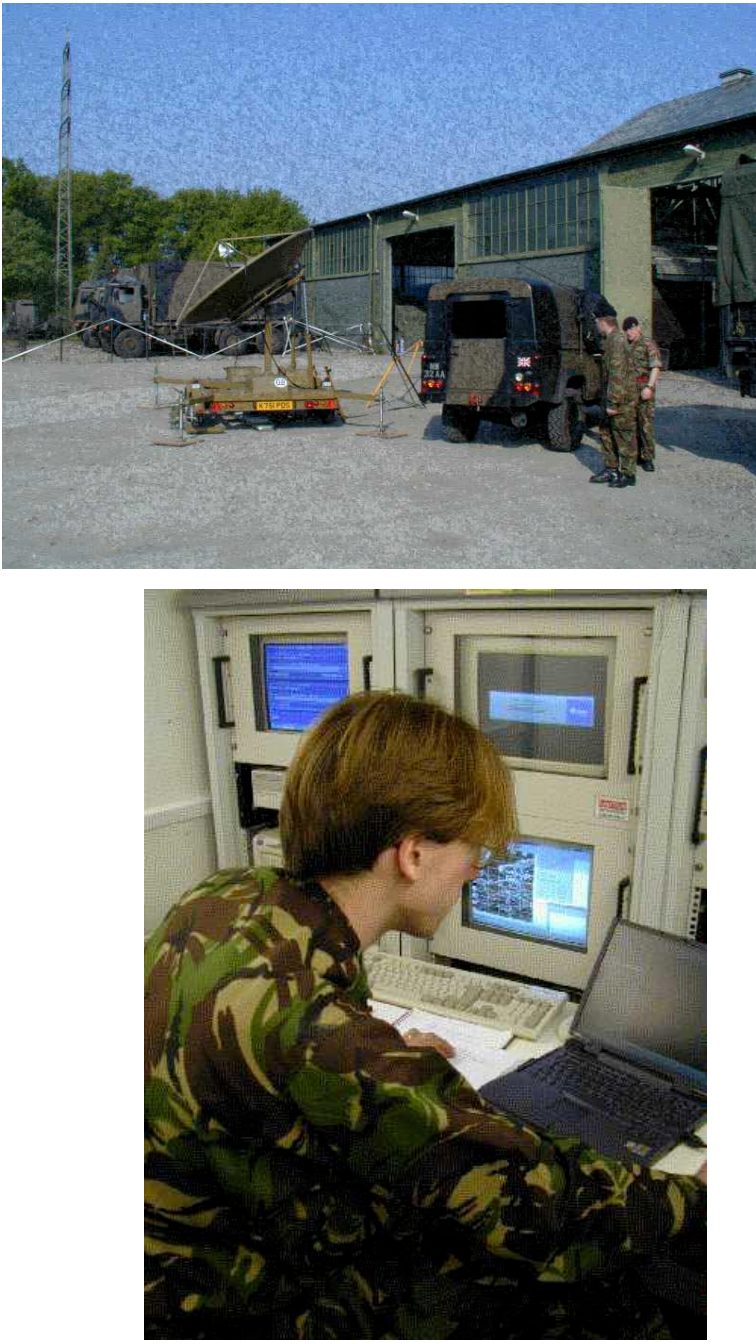

Fig 6 Mobile ground segment in use 


\section{Meeting the UK national needs}

As stated in the introduction, Topsat has been funded through two separate UK government agencies $-\mathrm{MoD}$ and BNSC. The proposal was originally made to the MoD to provide practical information on the usability of small satellites and the practical implications of using small, commandable assets in a tactical environment. Through the Topsat programme this objective is already being progressed, as can be seen in the images comprising Figure 6, where the mobile ground station is seen on exercise with UK forces in Germany in May 2001.

BNSC funding was won through a competitive process where the evaluation criteria were to demonstrate how the programme would advance the capability of and market for the UK small satellite industry. It is expected that this will be primarily achieved through the sale of similar systems, both with high resolution remote sensing payloads and with other payloads requiring the flexible but stable performance of the Topsat spacecraft.

\section{$\underline{\text { Status }}$}

The initial design work on the spacecraft and data handling system has been completed. The engineering model camera structure is complete and awaiting delivery of the complex off-axis mirrors, due in July 2001. Requests for proposals have been issued to launch providers to select the most appropriate and affordable launch. Early launch negotiation is also critical to enable the minimum required level of testing to be undertaken on the complex and sensitive camera structure. The camera structure has been vibrated to assess the modes and a validated model is being integrated with the spacecraft model to ensure appropriate unit level tests to be undertaken.

Launch is planned for late 2003, with a one-year period of operations to undertake the demonstrations required for $\mathrm{MoD}$ and further commercial exploitation of the system.
The outstanding critical issues in the programme are the validation of the camera optical and structural design and the qualification of the spacecraft structure. The ADCS which provides the other significant challenge has been simulated and appears feasible but can not be truly tested without an in-orbit demonstration.

\section{Acknowledgements}

Topsat is funded by the UK Ministry of Defence and the BNSC Mosaic small satellite initiative. Topsat is a collaborative programme between DERA, SSTL, RAL and Infoterra. The author wishes to thank Nigel Morris at RAL for information on and images of the camera and Jenny Harrison at SSTL for information on and images of the spacecraft. 